\title{
Efeito agudo dos exercícios de flexibilidade no desempenho de força máxima e resistência de força de membros inferiores e superiores
}

\author{
Anderson Caetano Paulo 1, 2, 3 \\ Carlos Ugrinowitsch ${ }^{2}$ \\ Gerson dos Santos Leite ${ }^{1}$ \\ Gisela Arsa ${ }^{1}$ \\ Paulo Henrique Marchetti 1, 4,5 \\ Valmor Tricoli $^{2}$ \\ ${ }^{1}$ Curso de Educação Física, Universidade Nove de Julho, São Paulo, SP, Brasil \\ ${ }^{2}$ Escola de Educação Física e Esporte, Departamento de Esportes, \\ USP - Universidade de São Paulo, São Paulo, SP, Brasil \\ ${ }^{3}$ Curso de Educação Física, UNIP - Universidade Paulista, São Paulo, SP, Brasil \\ ${ }^{4}$ Departamento de Educação Física, FEFISO - \\ Faculdade de Educação Física de Sorocaba, Sorocaba, SP, Brasil \\ ${ }^{5}$ Departamento de Educação Física, UNIMEP - Universidade Metodista de Piracicaba, SP, Brasil
}

Resumo: Este estudo verificou o efeito agudo dos exercícios de flexibilidade estática (EFlex) no desempenho de força máxima (FM) e de resistência de força (RF) em membros inferiores e superiores. Treze voluntários participaram do estudo e foram submetidos a testes de $\mathrm{FM}$ e RF (70\% $1 \mathrm{RM})$ nos exercícios supino e agachamento precedidos ou não de EFlex. O teste $T$ pareado foi utilizado para comparação das médias nas duas condições. Os EFlex diminuíram a FM no agachamento $(141,2 \pm 34,2$ vs $132 \pm 34,9 \mathrm{~kg} ; \mathrm{p}=0,007)$ e no supino $(77,5 \pm 21,7 \mathrm{vs} 71,7 \pm 17,7 \mathrm{~kg} p=0,04)$. A RF no agachamento não sofreu efeito dos EFlex $(16,2 \pm 5,7$ vs $16,3 \pm 6,8$ repetições $p=0,48)$, porém, no supino a $R F$ apresentou diminuição significante $(11,7 \pm 4,8$ vs $9,9 \pm 5,1$ repetições; $p=0,008)$. Portanto, os EFlex reduziram a FM nos membros inferiores e superiores e a RF somente nos membros superiores. Essa diferença na RF estaria relacionada ao volume de exercícios de flexibilidade pelo tamanho do grupo muscular.

Palavras-chave: performance; alongamento; treinamento físico.

\section{Acute effect of stretching exercises on upper and lower limbs maximum strength and strength endurance performance}

\begin{abstract}
This study investigated the acute effect of static stretching exercises (SSE) on maximum strength (MS) and strength endurance (SE) performance in lower and upper limbs. Thirteen volunteers participated in the study and were submitted to MS and SE (70\% 1RM) tests in the bench press and squat exercises with or without SSE. The paired T test showed that the SSE decreased MS in the squat $(141.2 \pm 34.2 \mathrm{vs} 132 \pm 34.9 \mathrm{~kg}$, $\mathrm{p}=0.007)$ and in the BP $(77.5 \pm 21.7$ vs $71.7 \pm 17.7 \mathrm{~kg} p=0.04)$. Squat SE was not affected by SSE $(16.2 \pm 5.7 \mathrm{vs}$ $16.3 \pm 6.8$ repetitions $\mathrm{p}=0.48)$. On the other hand, bench press SE decreased significantly after SSE (11.7 \pm 4.8 vs $9.9 \pm 5.1$ repetitions $p=0.008$ ). Therefore, SSE impaired MS performance on upper and lower limbs but SE was affected only on upper limbs. This difference in SE may be related to the stretching exercises volume applied to the size of each muscle group.
\end{abstract}

Keywords: performance; stretching; physical training.

\section{Introdução}

A flexibilidade e a força são importantes componentes dos programas de treinamento físico voltados para a saúde, qualidade de vida e desempenho esportivo (POLLOCK et al. 1998; $\underline{\mathrm{CHEN}}$ et al. 2010). É comum, na prescrição do treinamento físico-esportivo, a utilização de sessões complexas onde exercícios de flexibilidade e de força são combinados. Portanto, o entendimento da influência de uma capacidade motora sobre a outra é fundamental para a correta prescrição de exercícios evitando, dessa forma, os possíveis efeitos deletérios que possam influenciar 0 desempenho da atividade subsequente. 
Os efeitos agudos dos exercícios de flexibilidade no desempenho de testes de força máxima e de potência são encontrados na literatura e apresentam resultados controversos (RUBINI et al. 2007). Alguns estudos mostram melhora no desempenho desses testes (YAMAGUCHI e ISHII, 2005; LITTLE e WILLIAMS, 2006; MCMILLIAN et al., 2006; MANOEL et al., 2008), outros demonstram uma diminuição (NELSON et al., 2001; TRICOLI e PAULO, 2002; FLETCHER e JONES, 2004; CRAMER et al., 2005; WALLMANN et al., 2005; YAMAGUCHI et al., 2006; WINKE et al., 2010) ou mesmo nenhuma alteração ( $\underline{\mathrm{CHURCH}}$ et al., 2001; NOGUEIRA et al., 2010).

De modo semelhante, os três estudos encontrados que verificaram 0 efeito dos exercícios de flexibilidade no desempenho em testes de resistência de força (NELSON et al. 2005; FRANCO et al., 2008; GOMES et al., 2010) apresentaram resultados controversos. Nelson et al. (2005) encontraram um efeito negativo no desempenho do teste de resistência de força quando precedido de exercícios de flexibilidade estática. Já os outros dois estudos não observaram diferenças no resultado do teste de resistência de força quando precedido de exercícios de flexibilidade pelo método estático (FRANCO et al., 2008; GOMES et al., 2010).

Além dos resultados controversos, observa-se uma limitação em reproduzir os protocolos de exercícios de flexibilidade e de força utilizados nos estudos no campo da aplicação prática (FOWLES et al., 2000). Além disso, pouco se sabe a respeito do efeito dos exercícios de flexibilidade sobre a força e a resistência de força comparando membros superiores e inferiores. Parece que os músculos desses dois segmentos corporais respondem de forma distinta às tarefas que exigem desempenho de força máxima e de resistência de força (FERREIRA et al., 2006;

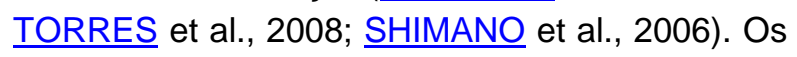
músculos dos membros inferiores são mais utilizados que os membros superiores no dia a dia, fato que o torna mais resistente a fadiga. Além disso, a diferença de tamanho e quantidade de unidades motoras entre os grupos musculares desses segmentos poderiam favorecer um melhor desempenho de força máxima e de resistência de força nos membros inferiores (SHIMANO et al., 2006).
Diante disso, o presente estudo teve por objetivo investigar os efeitos de uma sessão aguda de exercícios estáticos de flexibilidade no desempenho de força máxima e de resistência de força em membros inferiores e superiores.

\section{Materiais e métodos}

\section{Amostra}

Foram voluntários desse estudo treze homens, não atletas $(28,0 \pm 5,2$ anos; $86,7 \pm 15,9 \mathrm{~kg}$ e $179,5 \pm 8,0 \mathrm{~cm})$. Todos os sujeitos eram estudantes ou profissionais de educação física, fisicamente ativos, familiarizados com os exercícios de treinamento de força e com os exercícios de flexibilidade. Os participantes não possuíam histórico recente de lesões articulares, ósseas ou musculares nos membros superiores e inferiores e não faziam uso de suplementos alimentares ou medicamentos no momento do estudo. Todos participantes foram informados sobre os riscos e benefícios do estudo e assinaram consentimento informado antes da participação. O estudo foi previamente aprovado pelo Comitê de Ética em Pesquisa sob Protocolo n. 249996.

\section{Procedimentos}

O Quadro 1 sintetiza a sequência dos procedimentos experimentais que constaram de duas fases. Na primeira fase do estudo avaliou-se a influência dos exercícios de flexibilidade estática no desempenho de força máxima. Para isso, os voluntários realizaram o teste de força dinâmica máxima (1RM) nos exercícios agachamento e supino, que foram ou não precedidos de exercícios de flexibilidade de forma aleatória. Já a segunda fase verificou-se a influência dos exercícios de flexibilidade no desempenho de resistência de força. Nesta segunda fase os voluntários foram submetidos a um teste de repetições voluntárias máximas a 70\% $1 \mathrm{RM}$ até a falha concêntrica nos exercícios agachamento e supino, também precedidos ou não de exercícios de flexibilidade de forma aleatória. Os procedimentos para a realização dos testes de $1 \mathrm{RM}$ e de repetições máximas se basearam nas orientações da American Society of Exercise Physiologists (BROWN e WEIR, 2001). 
Quadro 1. Sequência dos procedimentos para as condições experimentais da fase 1 (força máxima) e da fase 2 (resistência de força) do estudo.

\begin{tabular}{|c|c|c|c|}
\hline \multicolumn{2}{|c|}{ Força Máxima (1RM) } & \multicolumn{2}{|c|}{ Resistência de Força (70\%1RM) } \\
\hline 1RM_s/FLEX & 1RM_c/FLEX & 70\%1RM_s/FLEX & 70\%1RM_c/FLEX \\
\hline \multicolumn{2}{|c|}{$5 \mathrm{~min}$ de corrida a $9 \mathrm{~km} / \mathrm{h}$} & \multicolumn{2}{|c|}{$5 \mathrm{~min}$ de corrida a $9 \mathrm{~km} / \mathrm{h}$} \\
\hline \multicolumn{2}{|c|}{ Teste flexibilidade do quadril } & \multicolumn{2}{|c|}{ Teste de flexibilidade do quadril } \\
\hline Repouso de 18min & $\begin{array}{c}\text { Exercícios de } \\
\text { flexibilidade do } \\
\text { quadril por } 18 \mathrm{~min}\end{array}$ & Repouso de 18min & $\begin{array}{c}\text { Exercícios de } \\
\text { flexibilidade do quadril } \\
\text { por } 18 \mathrm{~min}\end{array}$ \\
\hline \multicolumn{2}{|c|}{ Re-teste flexibilidade do quadril } & \multicolumn{2}{|c|}{ Re-teste flexibilidade do quadril } \\
\hline \multicolumn{2}{|c|}{ Teste 1RM no agachamento } & \multicolumn{2}{|c|}{ Teste $70 \% 1 \mathrm{RM}$ no agachamento } \\
\hline \multicolumn{2}{|c|}{$10 \mathrm{~min}$ de repouso } & \multicolumn{2}{|c|}{$10 \mathrm{~min}$ de repouso } \\
\hline \multicolumn{2}{|c|}{ Teste de flexibilidade do ombro } & \multicolumn{2}{|c|}{ Teste de flexibilidade do ombro } \\
\hline Repouso de 18min & $\begin{array}{l}\text { Exercícios de } \\
\text { flexibilidade do } \\
\text { Ombro por } 18 \mathrm{~min}\end{array}$ & Repouso de 18min & $\begin{array}{c}\text { Exercícios de } \\
\text { flexibilidade do Ombro } \\
\text { por } 18 \mathrm{~min}\end{array}$ \\
\hline \multicolumn{2}{|c|}{ Re-teste flexibilidade do ombro } & \multicolumn{2}{|c|}{ Re-teste flexibilidade do ombro } \\
\hline \multicolumn{2}{|c|}{ Teste 1RM no supino } & \multicolumn{2}{|c|}{ Teste $70 \% 1 \mathrm{RM}$ no supino } \\
\hline
\end{tabular}

Fase 1 - Avaliação da influência dos exercícios de flexibilidade estática no desempenho de força dinâmica máxima

Antes do início do estudo, os participantes foram submetidos a duas sessões de familiarização nas quais foram realizadas as estimativas das cargas de 1RM nos exercícios agachamento e supino realizados no aparelho Smith machine. Os valores máximos obtidos nestas familiarizações foram utilizados como carga inicial nas condições experimentais subsequentes.

Para condição experimental sem exercícios de flexibilidade para o teste de $1 \mathrm{RM}$ no exercício agachamento (Quadro 1), os sujeitos realizaram um aquecimento geral composto de $5 \mathrm{~min}$ de corrida a $9 \mathrm{~km} / \mathrm{h}$ em esteira ergométrica seguido por um teste de flexibilidade de extensão de quadril. Em seguida, eles permaneceram em repouso por $18 \mathrm{~min}$ e o re-teste da extensão de quadril foi realizado. A seguir iniciou-se 0 aquecimento especifico, composto de uma série de 5 repetições a $50 \% 1 \mathrm{RM}$ e outra de 3 repetições a $70 \% 1 \mathrm{RM}$ da carga estimada na familiarização do exercício agachamento. A execução do exercício agachamento teve início com os joelhos em completa extensão. No ponto intermediário do ciclo de movimento os joelhos deveriam atingir $90^{\circ}$ de flexão para então iniciar sua fase de extensão. $O$ ciclo de movimento foi finalizado com os joelhos novamente estendidos. Para a garantia dessa amplitude e segurança dos sujeitos foram utilizadas plataformas de madeiras ajustadas para uma altura equivalente a $90^{\circ}$ de flexão dos joelhos.

$\mathrm{Na}$ condição experimental sem exercícios de flexibilidade para o teste de 1RM no exercício supino, os procedimentos foram similares ao do teste de 1RM no agachamento. Dez minutos após o teste de 1RM do agachamento realizou-se um teste de flexibilidade para abdução do ombro. Após permanecerem $18 \mathrm{~min}$ em repouso os sujeitos refizeram o teste de abdução do ombro. Trinta minutos após o término do teste de $1 \mathrm{RM}$ do agachamento, os sujeitos foram submetidos ao aquecimento específico e ao teste de 1 RM no supino. Para realizar 0 teste, 0 voluntário permaneceu deitado no banco de supino e manteve os pés em contato com o solo. $\mathrm{O}$ ciclo de movimento do exercício iniciou com extensão completa dos cotovelos, e em seguida a barra deveria descer (flexão dos cotovelos) até encostar o terço superior do esterno e então retornar à posição inicial.

Os procedimentos da condição com exercícios de flexibilidade foram iguais às condições experimentais anteriores, mas com a inclusão de $18 \mathrm{~min}$ de exercícios de flexibilidade para os membros inferiores ou superiores, antes da execução do agachamento ou do supino, respectivamente, conforme descrição no Quadro 1.

Fase 2 - Avaliação da influência dos exercícios de flexibilidade estática no desempenho de resistência de força

$\mathrm{Na}$ familiarização da fase 2 foi calculada uma carga fixa de $70 \%$ do maior valor obtido nos testes de 1RM nos exercícios supino e 
agachamento. A seguir o sujeito executou séries de repetições máximas até a falha concêntrica para ambos os exercícios.

Nessa fase as condições experimentais com e sem exercícios de flexibilidade foram idênticas as mesmas condições da fase 1 à exceção do tipo de teste de força (Quadro 1). Assim, os voluntários realizaram uma série do maior número de repetições voluntárias a $70 \% 1 \mathrm{RM}$ até falha concêntrica do movimento nos exercícios agachamento e supino nas condições com e sem a presença dos exercícios de flexibilidade.

\section{Exercícios de flexibilidade estática}

Para as condições experimentais com exercícios de flexibilidade foram escolhidos seis exercícios para os grupos musculares diretamente envolvidos na execução do agachamento (extensores de joelho e quadril, posteriores da coxa e adutores do quadril) e seis exercícios para grupos musculares diretamente envolvidos na execução do supino (adutores de ombro e extensor de cotovelo). Cada sujeito realizou três repetições de 30 segundos na posição estática com intervalos de 30 segundos entre as repetições. Isso totalizou 18 minutos de atividade por segmento corporal. A sensação de desconforto muscular na maior amplitude possível foi utilizada como parâmetro para regular a intensidade de cada exercício de flexibilidade.
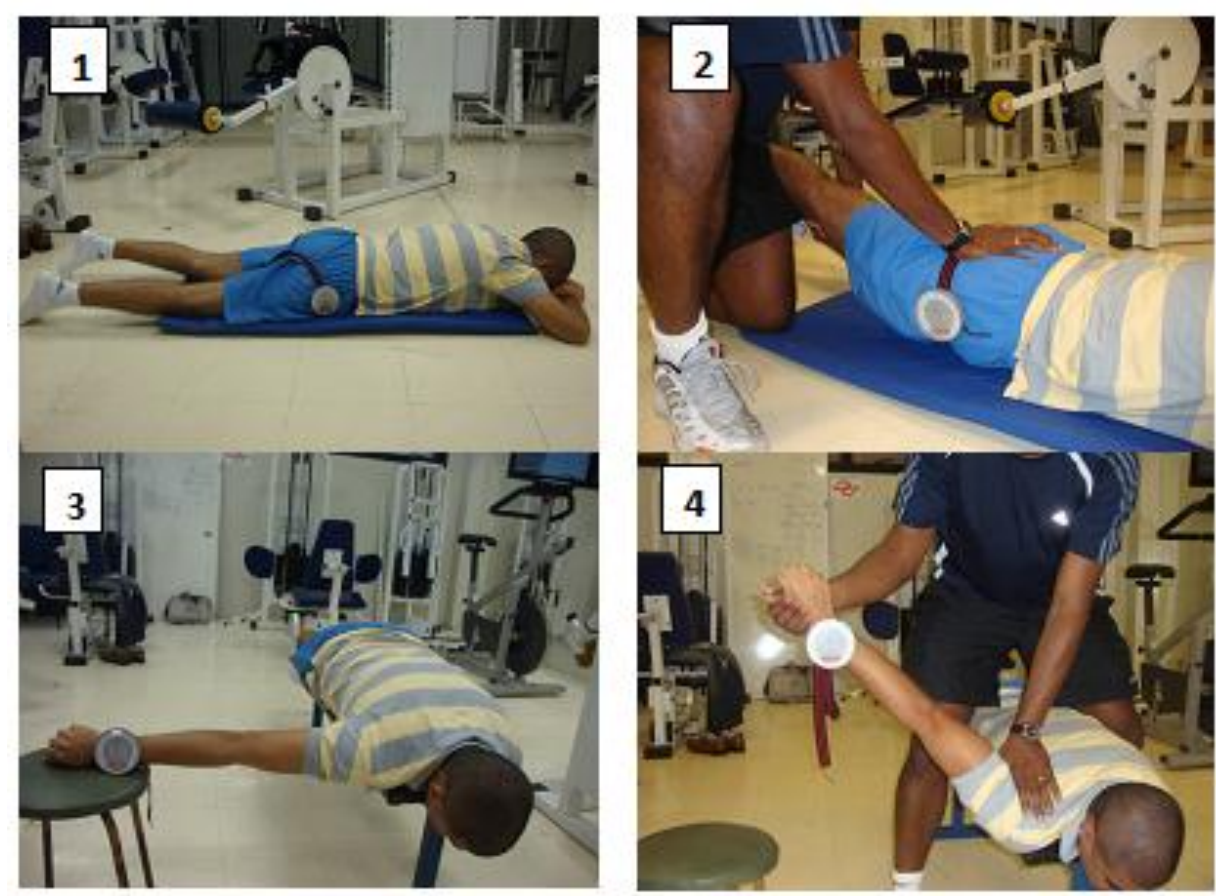

Figura 1. Posição inicial e final dos testes de flexibilidade da extensão de quadril (fotos 1 e 2 ) e da abdução do ombro (fotos 3 e 4 ). 


\section{Análise estatística}

Os resultados estão apresentados com estatística descritiva (média \pm desvio padrão). A normalidade e homogeneidade dos dados foram verificadas utilizando o teste de Shapiro Wilk.

Para comparar a flexibilidade ao redor das articulações do quadril e do ombro (teste e reteste) nas condições com e sem flexibilidade utilizou-se a análise de variância para medidas repetidas (ANOVA two way) [fator teste $(2 \mathrm{x}) \mathrm{x}$ fator condição $(2 x)$ ]. Na ocorrência de valores $F$ significantes foi utilizado o post-hoc de Fisher.

Utilizou-se o teste $T$ de student para comparar as médias dos valores obtidos no teste de 1RM (fase 1) e no teste de resistência de força (fase 2) entre as condições com e sem exercícios de flexibilidade nos exercícios agachamento e supino. O nível de significância adotado foi $\mathrm{p}<0,05$.

Em adição, o tamanho do efeito foi calculado (RHEA, 2004) para a alteração do desempenho de força dinâmica máxima, da resistência de força e para a alteração na amplitude articular.

\section{Resultados}

A Anova identificou que a amplitude de extensão do quadril aumentou $12,9 \%(p=0,03)$ e a de abdução do ombro aumentou 9,4\% $(p=0,04)$ após a execução dos exercícios de flexibilidade (Figura 2). Isso resultou em tamanhos de efeito de 0,45 e 0,53 , respectivamente.

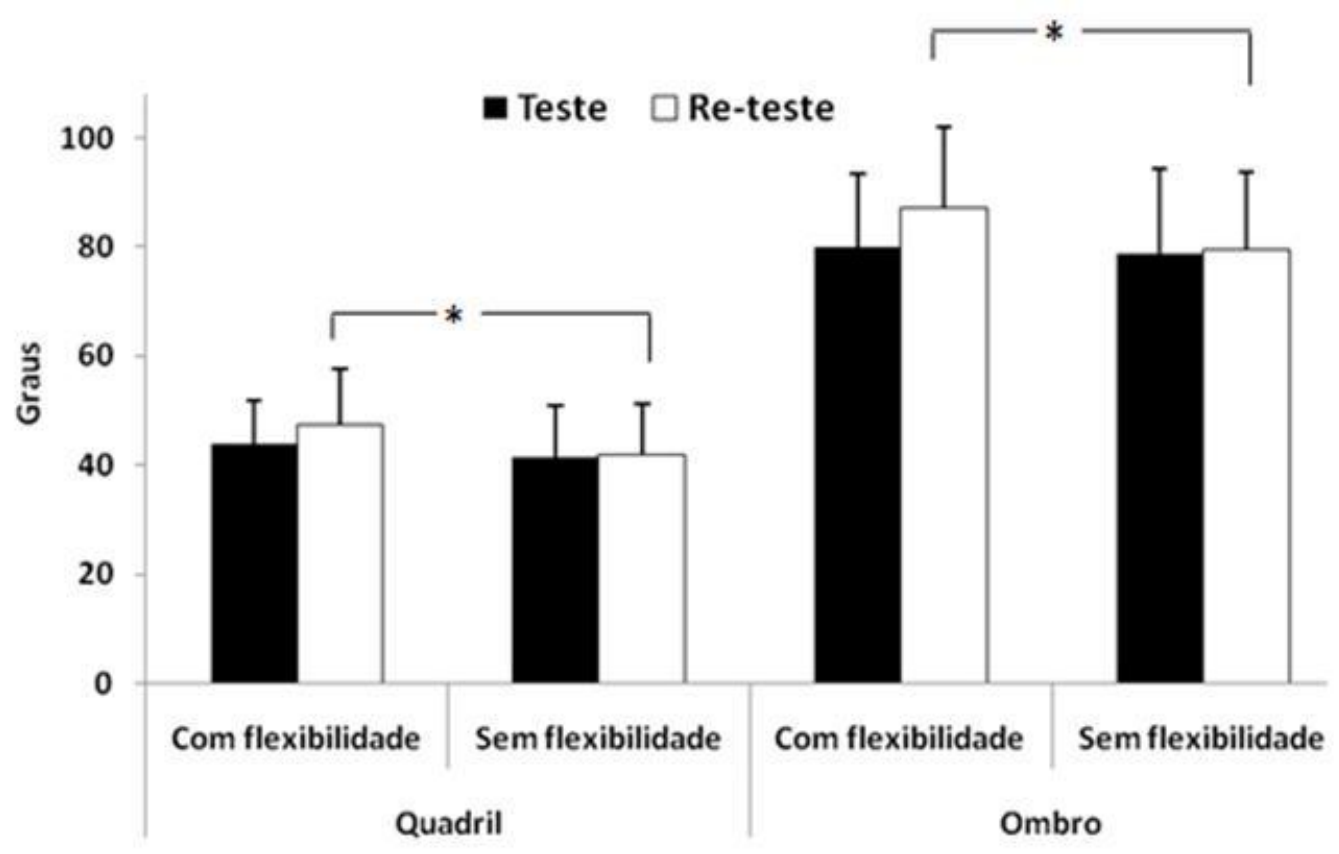

Figura 2. Valores médios ( \pm desvio padrão) de ângulo articular de extensão do quadril e de abdução de ombro nas condições com e sem os exercícios de flexibilidade. *valor com flexibilidade maior que sem flexibilidade intra-articulação $(p<0,05)$.

No desempenho de força máxima foi verificada diferença estatisticamente significante no teste de $1 \mathrm{RM}$ entre as condições com e sem flexibilidade nos exercícios agachamento e supino. A presença dos exercícios de flexibilidade estática reduziu o desempenho de força máxima em $7,0 \%$ no agachamento $(p=0,007)$ e em $8,1 \%$ no supino $(p=0,04)$.

O desempenho no teste de repetições máximas a $70 \% 1 \mathrm{RM}$ foi afetado apenas no exercício supino. $\mathrm{Na}$ condição com flexibilidade houve uma diminuição percentual do número de repetições de $18,2 \%$ ( $p=0,008)$, enquanto que no agachamento os resultados se mantiveram inalterados $(p=0,48)$.

O cálculo do tamanho do efeito para o teste de $1 \mathrm{RM}$ nos exercícios agachamento e supino foram respectivamente 0,27 e 0,27 . Já o cálculo para o teste de resistência de força foi de 0,02 e 0,38 para os exercícios agachamento e supino, respectivamente. 
Tabela 1. Desempenho de força máxima $(\mathrm{kg})$ e de resistência de força (repetições) nos exercícios agachamento e supino com e sem exercícios de flexibilidade.

\begin{tabular}{cccccc}
\hline & \multicolumn{2}{c}{ 1RM } & & \multicolumn{2}{c}{ Resistência de Força } \\
\cline { 2 - 3 } \cline { 5 - 6 } Condição & Agachamento $(\mathrm{kg})$ & Supino $(\mathrm{kg})$ & & Agachamento (rep) & Supino (rep) \\
\cline { 2 - 3 } \cline { 5 - 6 } Sem flexibilidade & $141,2 \pm 34,2$ & $77,5 \pm 21,7$ & & $16,2 \pm 5,7$ & $11,7 \pm 4,8$ \\
Com flexibilidade & $132,0 \pm 34,9^{*}$ & $71,7 \pm 17,7^{*}$ & & $16,3 \pm 6,8$ & $9,9 \pm 5,1^{*}$ \\
\hline
\end{tabular}

${ }^{*}$ Condição com flexibilidade menor que condição sem flexibilidade intra-exercício $(p<0,05)$.

\section{Discussão}

O objetivo desse estudo foi verificar o efeito agudo de exercícios de flexibilidade estática no desempenho de força máxima e de resistência de força em membros inferiores e superiores. Os principais achados foram que os exercícios de flexibilidade diminuíram o desempenho de força máxima nos membros superiores (supino) e inferiores (agachamento) enquanto que a resistência de força foi diminuída somente nos membros superiores. Isso indica que 0 desempenho de resistência de força não foi afetado de maneira idêntica ao da força máxima quando antecedidos de exercícios de flexibilidade estática. Nossos resultados estão em acordo com a literatura que reporta a diminuição do desempenho no teste de $1 \mathrm{RM}$ quando precedido de exercícios de flexibilidade estática tanto em membros inferiores (TRICOLI e PAULO, 2002; BACURAU et al., 2009; WINCHESTER et al., 2009) quanto em superiores (EVETOVICH et al., 2003).

Os exercícios de flexibilidade aplicados neste estudo aumentaram a amplitude articular da extensão do quadril e da abdução do ombro. Isso mostra que os exercícios aplicados foram efetivos para alterar a flexibilidade de forma aguda. Esse efeito agudo pode estar relacionado a uma diminuição da tensão viscoelástica ou alterações de respostas reflexas (TAYLOR et al. 1990) os quais podem explicar nossos principais achados.

De fato, Bacurau et al. (2009), também atribuíram a diminuição do desempenho no teste de 1RM ao aumento da amplitude articular dos membros inferiores, após a aplicação do método estático de flexibilidade em mulheres fisicamente ativas e estudantes de educação física. Porém, estes pesquisadores encontraram uma diminuição de $13,4 \%$ no desempenho de força dinâmica máxima. Assim, a diferença entre gêneros parece ser uma das variáveis intervenientes que influencia nos efeitos dos exercícios de flexibilidade. As mulheres são mais flexíveis (DE ARAUJO, 2008) e respondem de forma diferente no desempenho de flexibilidade quando submetidas a um mesmo estímulo de treinamento (FELAND et al., 2010). Parece que os homens são menos suscetíveis aos efeitos agudos da flexibilidade, pois no estudo do Bacurau et al. (2009) também foram realizados 3 repetições de 6 exercícios de flexibilidade estática (30s exercício:30s pausa) totalizando um volume de exercícios similar ao nosso estudo.

Diferença no volume dos exercícios de flexibilidade pode afetar de maneira diferenciada a redução da força. Molacek et al. (2010) não encontraram diferença no desempenho de força máxima no exercício supino quando precedido de 4 repetições (2 exercícios) do método estático ou da facilitação neuromuscular proprioceptiva (FNP). Porém, no presente estudo, foi aplicado um total de 18 repetições por segmento corporal (3 repetições de 6 exercícios). A intensidade do exercício de flexibilidade também é uma variável que pode explicar a alteração no desempenho de força máxima. Molacek et al. (2010) relataram uma intensidade de $75 \%$ da percepção máxima de esforço durante os exercícios de flexibilidade estática. No nosso estudo os sujeitos atingiam a situação de desconforto máximo; portanto, a intensidade dos exercícios de flexibilidade estática foi maior. De fato, Winchester et al. (2009) demonstraram em seu estudo que com apenas uma única série de 30 segundos realizada no desconforto máximo, houve uma redução de $5,4 \%$ no desempenho de força máxima dos músculos flexores do joelho.

Um dos mecanismos que poderia estar envolvido na diminuição da força máxima após os exercícios de flexibilidade seria a inabilidade de recrutar um maior número de unidades motoras devido à alta atividade reflexa inibitória durante o 
alongamento dos músculos (FOWLES et al., 2000). Decréscimos na ativação das unidades motoras têm sido mencionados através da avaliação da amplitude do sinal eletromiográfico e também da porcentagem de inativação da musculatura envolvida na tarefa (FOWLES et al., 2000; BEHM et al., 2001). Isso pode ser consequência da inibição provocada pelos órgãos tendinosos de Golgi que teriam sido estimulados pela tensão desenvolvida na unidade músculotendão durante as atividades de flexibilidade e também da contribuição reflexa dos receptores de dor. No entanto, esse mecanismo é controverso, pois há estudos que observaram redução no desempenho de força máxima com o método estático de flexibilidade sem haver alteração do sinal eletromiográfico (HERDA et al., 2008; ESPOSITO et al., 2009; EVETOVICH et al., 2003). Esses estudos apontam que a principal causa dessa redução seria em função dos aspectos mecânicos que comprometem o tempo para atingir $o$ pico de força, a taxa de desenvolvimento de força e até a aceleração do movimento. Parte dessas alterações mecânicas pode ser observada pela diminuição da rigidez tecidual (stiffness) que está associada à rigidez da musculatura esquelética, tecido conjuntivo, cápsula articular, tendões e ligamentos. Os exercícios de flexibilidade têm a capacidade de alterar as propriedades viscoelásticas da unidade músculo-tendão reduzindo a tensão passiva e a rigidez da unidade (EVETOVICH et al. 2003). Um sistema músculo-tendão mais maleável (menor stiffness) seria menos eficiente para a transmissão de força produzida pela musculatura esquelética para o sistema ósteo-articular (TRICOLI e PAULO, 2002).

Com relação ao desempenho de resistência de força, os resultados foram diferentes para os membros inferiores e superiores. Nos membros inferiores não houve influência dos exercícios de flexibilidade estática no número de repetições executadas no exercício agachamento; já para os membros superiores houve diminuição significante do desempenho de resistência de força. Nelson et al. (2005) mediram o desempenho de resistência de força dos músculos flexores do joelho em voluntários submetidos ao método estático de flexibilidade. Diferente do nosso estudo, os autores apontaram redução significante no número de repetições máximas no exercício flexão de joelho. Entretanto, os voluntários foram submetidos a oito repetições de dois exercícios de flexibilidade para os posteriores de coxa. Já no nosso estudo, as dezoito repetições de seis exercícios foram divididas entre extensores de joelho, extensores de quadril, posteriores de coxa e adutores do quadril. Além disso, pelo fato do exercício agachamento ser de característica multiarticular, talvez não tenhamos atingido um volume de exercícios necessário para afetar todos os músculos envolvidos neste movimento a fim de diminuir o desempenho de resistência de força. Outra hipótese para esse resultado no membro inferior seria o método de flexibilidade aplicado, pois, Franco et al. (2008) e Gomes et al. (2010) também não encontraram diferença estatística com o método estático. Nesses dois estudos houve um menor volume de exercícios de flexibilidade quando comparado ao estudo do Nelson et al. (2005).

Entretanto, diferentemente dos resultados encontrados para os membros superiores utilizando o método estático (FRANCO et al., 2008 e GOMES et al., 2010) nosso estudo encontrou uma redução no desempenho de resistência de força desse grupo muscular. Franco et al. (2008) e Gomes et al. (2010) utilizaram um e dois exercícios de flexibilidade, respectivamente, para realizar o supino enquanto no nosso estudo foram executados seis exercícios. Levando em consideração que existe uma menor quantidade de músculos envolvidos e o movimento é menos complexo que o agachamento, o volume mais elevado de exercícios pode ser uma explicação plausível para essa diferença nos resultados. Além disso, o membro superior apresenta uma menor resistência de força quando comparado ao membro inferior ( et al., 1990; SHIMANO et al., 2006). Uma resposta de controle neural que acontece durante um teste de resistência de força com cargas submáximas é o recrutamento de unidades motoras de forma assincrônica, pois diferentemente de um teste de força máxima, elas não precisam ser recrutadas simultaneamente. Esse mecanismo serve para atrasar o aparecimento da fadiga, pois permite que algumas fibras musculares descansem enquanto outras produzem força para manter a tarefa motora. Como os exercícios de flexibilidade podem ter alterado o padrão de recrutamento é possível que pelo fato de existir um menor número absoluto de unidades motoras nos 
membros superiores, houve um menor revezamento na ativação destas unidades resultando num menor tempo de descanso para as fibras musculares, o que ocasionou a diminuição do número de repetições devido à fadiga.

\section{Conclusão}

Podemos concluir que exercícios de flexibilidade estática, equalizados em volume e intensidade, comprometem mais o desempenho de resistência de força em músculos dos membros superiores do que dos membros inferiores. Parece que os músculos dos membros superiores são mais suscetíveis aos efeitos agudos dos exercícios de flexibilidade, devido à menor quantidade de unidades motoras e menor tamanho do grupo muscular.

\section{Referências}

BACURAU, R.F.; MONTEIRO, G.A.; UGRINOWITSCH, C.; TRICOLI, V.; CABRAL, L.F.; AOKI, M.S. Acute effect of a ballistic and a static stretching exercise bout on flexibility and maximal strength. Journal of Strength

Conditioning Research, Connecticut, v. 23, n. 1, p. 304-308, 2009. Disponível em:

$<$ http://journals.Iww.com/nscajscr/fulltext/2009/01000/acute effect of a ballistic and a static.43.aspx>. Acesso em: 23 mai. 2010.

BEHM, D.G.; BUTTON, D.C.; BUTT, J.C. Factors affecting force loss with prolonged stretching. Canadian Journal of Applied Physiology, Champaign, v.26, n. 3, p.262-272, 2001. Disponível em: $<$ http://www.ncbi.nlm.nih.gov/pubmed/11441230>. Acesso em: 27 mai. 2010.

BROWN, L. E., WEIR, J. P. Recommendation of procedures of the American Society of Exercise Physiologists (ASEP) I: evaluation of muscular strength and power. Journal of Exercise Physiology, Winston, v. 4, n. 3, p. 1-21, 2001. Disponível em: $<$ http://fullerton.academia.edu/LeeBrown/Papers/4 13272/ASEP Procedures Recommendation I Ac curate Assessment of Muscular Strength and Power>. Acesso em: 21 jun. 2010.

CHEN, C.H.; NOSAKA, K.; CHEN, H.L.; LIN, M.J.; TSENG, K.W.; CHEN, T.C. Effects of Flexibility Training On Eccentric Exercise-Induced Muscle Damage. Medicine and Science in Sports and Exercise, Indianapolis, v.43, n.3, 2010. Disponível em:
$<$ http://www.ncbi.nlm.nih.gov/pubmed/20689450>. Acesso em: 04 mai. 2011.

CHURCH, J. B.; WIGGINS, M. S.; MOODE, E. M.; CRIST, R. Effect of warm-up and flexibility treatments on vertical jump performance. Journal Strength Conditioning Research, Connecticut, v. 15, n. 3, p. 332-336, 2001. Disponível em: $<$ http://journals.lww.com/nscaiscr/Abstract/2001/08000/Effect of Warm Up an d Flexibility Treatments on.12.aspx $>$. Acesso em: 27 mai. 2010.

CRAMER, J.T.; HOUSH, T.J.; WEIR, J.P.; JOHNSON, G.O.; COBURN, J.W.; BECK, T.W. The acute effects of static stretching on peak torque, mean power output, electromyography, and mechanomyography. European Journal of Applied Physiology, Berlim, v. 93 n. 5-6, p. 530539, 2005. Disponível em:

$<$ http://www.springerlink.com/content/xald6t74ftye oy2j/>. Acesso em: 27 mai. 2010.

DE ARAÚJO, C.G.S. Avaliação da Flexibilidade: Valores Normativos do Flexiteste dos 5 aos 91 Anos de Idade. Arquivos Brasileiros de Cardiologia, Rio de Janeiro, v. 90, n.4, p. 280287, 2008. Disponível em: $<$ http://www.scielo.br/pdf/abc/v90n4/v90n4a08.pdf >. Acesso em: 27 mai. 2010.

ESPOSITO, F.; CÉ E.; RAMPICHINI, S.; VEICSTEINAS, A. Acute passive stretching in a previously fatigued muscle: Electrical and mechanical response during tetanic stimulation. Journal of Sports Sciences, Londres, v.27, n.12, p.1347-1357, 2009. Disdponível em:

$<$ http://www.blackwellpublishing.com/aphmeeting/ abstract.asp? MeetinglD $=760 \& i d=79430>$. Acesso em: 04 mai. 2011.

EVETOVICH, T.K.; NAUMAN, N.J.; CONLEY, D.S.; TODD, J.B. Effect of static stretching of the biceps brachii on torque, electromyography, and mechanomyography during concentric isokinetic muscle actions. Journal of Strength

Conditioning Research, Connecticut, v. 17, n. 3, p. 484-488, 2003. Disponível em: $<$ http://journals.Iww.com/nscaiscr/Abstract/2003/08000/Effect of Static Stretchi $\mathrm{ng}$ of the Biceps Brachii.9.aspx>. Acesso em: 04 mai. 2011.

FELAND, J.B.; HAWKS, M. HOPKINS, J.T.; HUNTER, I.; JOHNSON, A.W.; EGGETT, D.L. Whole body vibration as an adjunct to static stretching. International Journal of Sports Medicine. Stuttgart v.31, n. 8, p. 584-589, 2010. Disponível em: $<$ https://www.thiemeconnect.de/ejournals/html/sportsmed/doi/10.1055/ s-0030-1254084>. Acesso em: 04 mai. 2011. 
FERREIRA, S.F.; MARINS, J.C.B.; SILVA, L.C.; LUNZ, W.; PIMENTEL, G.G.A.; MIGLIORINI, E.M. Determinação de perfil de repetições máximas no exercício de extensão de pernas e supino reto com diferentes percentuais de força. Revista da Educação Física, Maringá, v. 17, n. 2, p. 149159, 2006. Disponível em: $<$ http://periodicos.uem.br/ojs/index.php/RevEducFi s/article/view/3335/2408>. Acesso em: 17 fev. de 2009.

FLETCHER, I.M.; JONES, B. The effect of different warm-up stretch protocols on 20 meter sprint performance in trained rugby union players. Journal of Strength Conditioning Research, Connecticut, v.18, n.4, p. 885-888, 2004. Disponível em: <http://journals.lww.com/nscaiscr/Abstract/2004/11000/The Effect of Different Warm Up Stretch Protocols.35.aspx>. Acesso em: 27 ago. 2009.

FOWLES, J.R.; SALE, G.; MACDOUGALL, J. D. Reduced strength after passive stretch of the human plantarflexors. Journal Applied

Physiology. Bethesda, v. 89, n.3, p. 1179-1188, 2000. Disponível em:

<http://jap.physiology.org/content/89/3/1179.full >. Acesso em: 01 jun. 2009.

FRANCO, B.L.; SIGNORELLI, G.R.; TRAJANO, G.S.; OLIVEIRA, C.G. Acute effects of different stretching exercises on muscular endurance. Journal of Strength Conditioning Research, Connecticut, v. 22, n.6, p. 1832-1837, 2008. Disponível em: <http://journals.Iww.com/nscajscr/Fulltext/2008/11000/Acute Effects of Differe nt Stretching Exercises on.16.aspx>. Acesso em: 13 set. 2010 .

GOMES, T.M.; SIMÃO, R.; MARQUES, M.C.; COSTA, P.B.; DA SILVA NOVAES, J. Acute Effects of Two Different Stretching Methods on Local Muscular Endurance Performance. Journal of Strength Conditioning Research, Connecticut, v. 25, n.3, p. 745- 752, 2011. Disponível em: <http://journals.Iww.com/nscaiscr/Fulltext/2011/03000/Acute Effects of Two D ifferent Stretching Methods.23.aspx>. Acesso em: 20 nov. 2011.

HERDA, T.J.; CRAMER, J.T.; RYAN, E.D.; MCHUGH, M.P.; STOUT, J.R.; Acute effects of static versus dynamic stretching on isometric peak torque, electromyography, and mechanomyography of the biceps femoris muscle. Journal of Strength Conditioning Research, Connecticut, v. 22, n.3, p. 809-817, 2008. Disponível em: <http://journals.Iww.com/nscajscr/Fulltext/2008/05000/Acute Effects of Static versus Dynamic Stretching.23.aspx>. Acesso em: 20 set. 2010 .
HOEGER, W.W.K.; HOPKINS, D.R.; BARETTE, S.L.; HALE, D.F. Relationship between repetitions and selected percentages of one repetition maximum: A comparison between untrained and trained males and females. Journal of Applied

Sport Science Research, Connecticut, v.4, n. 2, p.:47-54. 1990. Disponível em:

$<$ http://journals.Iww.com/nsca-

jscr/Abstract/1990/05000/Relationship between Repetitions and Selected.4.aspx>. Acesso em: 13 out. 2009.

HOEGER, W.W.K.; BARETTE, S.L.; HALE, D.F.; HOPKINS, D.R. Relationship between repetitions and selected percentages of one repetition maximum. Journal of Applied Sport Science Research, Connecticut, v.1, n.1, p.11-13. 1987. Disponível em: <http://journals.lww.com/nscajscr/Abstract/1987/02000/Relationship Between Repetitions and Selected.2.aspx>. Acesso em: 13 out. 2009.

LITTLE, T.; WILLIAMS, A.G. Effects of differential stretching protocols during warm-ups on highspeed motor capacities in professional soccer players. Journal of Strength Conditioning Research, Connecticut, v.20, n.1, 203-207, 2006. Disponível em: <http://journals.lww.com/nscaiscr/Abstract/2006/02000/Effects of Differential S tretching Protocols.33.aspx>. Acesso em: 17 out. 2010.

MANOEL, M.E.; HARRIS-LOVE, M.O.; DANOFF, J.V.; MILLER, T.A. Acute effects of static, dynamic, and proprioceptive neuromuscular facilitation stretching on muscle power in women. Journal of Strength Conditioning Research, Connecticut, v.22, n.5, p.1528-1534, 2008. Disponível em: <http://journals.lww.com/nscajscr/Fulltext/2008/09000/Acute Effects of Static, Dynamic, and.19.aspx>. Acesso em: 27 ago. 2009.

McMILLIAN, D.J.; MOORE, J.H.; HATLER, B.S.; TAYLOR, D.C. Dynamic vs. static-stretching warm up: the effect on power and agility performance. Journal of Strength Conditioning Research, Connecticut, v.20, n.3, p. 492-499, 2006. Disponível em: <http://journals.lww.com/nscajscr/Abstract/2006/08000/Dynamic Vs Static Stre tching Warm Up the Effect.6.aspx>. Acesso em: 03 fev. 2010.

MOLACEK, Z.D.; CONLEY, D.S.; EVETOVICH, T.K.; HINNERICHS, K.R. Bench press performance in collegiate football players. Journal of Strength Conditioning Research,

Connecticut, v. 24, n.3, p. 711-716, 2010.

Disponível em: <http://journals.lww.com/nscajscr/Fulltext/2010/03000/Effects of Low and $\mathrm{Hi}$ gh Volume Stretching on.17.aspx>. Acesso em: 22 dez. 2010. 
NELSON, A.G.; KOKKONEN, J.; ARNALL, D.A. Acute muscle stretching inhibits muscle strength endurance performance Journal of Strength Conditioning Research, Connecticut, v.19, n.2, p. 338-343, 2005. Disponível em:

$<$ http://journals.Iww.com/nscajscr/Abstract/2005/05000/Acute Muscle Stretchin g Inhibits Muscle Strength.17.aspx>. Acesso em: 14 jun. 2009.

NELSON, A.G.; GUILLORY, I.K.; CORNWELL, C.; KOKKONEN, J. Inhibition of maximal voluntary isokinetic torque production following stretching is velocity-specific. Journal of Strength

Conditioning Research, Connecticut, v.15, n.2, p. 241-246, 2001.Disponível em:

$<$ http://journals.Iww.com/nsca-

jscr/Abstract/2001/05000/Inhibition of Maximal V oluntary Isokinetic Torque.14.aspx>. Acesso em: 14 jun. 2009.

NOGUEIRA, C.J.; GALDINO, L.A.S.; VALE, R.G.S.; DANTAS, E.H.M. Efeito agudo do alongamento estático sobre o desempenho no salto vertical, Motriz. Revista de Educação Física. UNESP, Rio Claro, v.16, n.1, p.10-16, 2010. Disponível em:

$<$ http://www.periodicos.rc.biblioteca.unesp.br/inde x.php/motriz/article/view/1980-

6574.2010v16n1p10 >. Acesso em: 22 dez. 2010.

POLLOCK, M. L.; GAESSER, G. A.; BUTCHER, J. D.; DESPRES, J. P.; DISHMAN, R. K.; FRANKLIN, B. A., et al. ACSM position stand on the recommended quantity and quality of exercise for developing and mantaining cardiorespiratory and muscular fitness, and flexibility in healthy adults. Medicine and Science in Sports and Exercise, Indianapolis, v.30, n.6, p. 975-991, 1998. Disponível em:

$<$ http://journals.lww.com/acsm-

msse/Abstract/1998/06000/ACSM Position Stand

The Recommended Quantity and.32.aspx>.

Acesso em: 20 set. 2010.

RHEA, M.R. Determining the Magnitude of Treatment Effects in Strength Training Research Through the Use of Effect Size. Journal of Strength Conditioning Research, Connecticut, v.18, n.4, p. 918-920, 2004. Disponível em: $<$ http://journals.lww.com/nscajscr/Abstract/2004/11000/Determining the Magnit ude of Treatment Effects in.40.aspx>. Acesso em: 22 mar. 2012.

RUBINI, E.C; COSTA, A.L.L.; GOMES, P.S.C. The effects of stretching on strength performance. Sports Medicine, Auckland, v. 37, n. 3, p. 213224, 2007. Disponível em:

$<$ http://adisonline.com/sportsmedicine/Abstract/20 $07 / 37030 /$ The Effects of Stretching on Strength Performance.3.aspx>. Acesso em: 29 mai. 2010.
SHIMANO, T.; KRAEMER, W.J.; SPIERING, B.A.; VOLEK, J.S.; HATFIELD, D.L.; SILVESTRE, R.; VINGREN, J.L.; FRAGALA, M.S.; MARESH, C.M.; FLECK, S.J.; NEWTON, R.U.;

SPREUWENBERG, L.P.; HÄKKINEN K.

Relationship between the number of repetitions and selected percentages of one repetition maximum in free weight exercises in trained and untrained men. Journal of Strength

Conditioning Research. Connecticut, v. 20, n.4, p. 819-823, 2006. Disponível em:

$<$ http://journals.Iww.com/nscaiscr/Abstract/2006/11000/Relationship Between t he Number of Repetitions and.15.aspx>.

Acesso em: 12 mai. 2011.

TAYLOR, D.C.; DALTON, J.D.; SEABER, A.V.; GARRETT,W.E. Viscoelastic properties of muscle-tendon units: the biomechanical effects of stretching. American Journal of Sports

Medicine, Baltimore, v.18, n. 3, p. 300-309, 1990. Disponível em:

$<$ http://ajs.sagepub.com/content/18/3/300.abstract >. Acesso em: 10 out. 2011.

TORRES, E.M.; KRAEMER, W.J.; VINGREN, J.L.; VOLEK, J.S.; HATFIELD, D.L.; SPIERING, B.A.; HO, J.Y.; FRAGALA, M.S.; THOMAS, G.A.; ANDERSON, J.M.; HÄKKINEN, K.; MARESH, C.M. Effects of stretching on upper-body muscular performance. Journal of Strength Conditioning Research, Connecticut, v.22, n. 4, p. 1279-1285, 2008. Disponível em:

$<$ http://journals.Iww.com/nsca-

jscr/Fulltext/2008/07000/Effects of Stretching on Upper Body Muscular.35.aspx>. Acesso em: 15 ago. 2011.

TRICOLI, V; PAULO, A.C. Efeito agudo dos exercícios de alongamento sobre o desempenho da força máxima. Revista de Atividade Física \& Saúde, Londrina, v. 7, n1, p.06-13, 2002. Disponível em: <http://bases.bireme.br/cgibin/wxislind.exe/iah/online/?IsisScript=iah/iah.xis\& src $=$ google\&base $=$ LILACS\&lang $=$ p\&nextAction $=$ In k\&exprSearch=314630\&indexSearch=ID >. Acesso em: 14 fev. 2009.

WALLMANN, H.W.; MERCER, J.A.; MCWHORTER, J.W. Surface electromyographic assessment of the effect of static stretching of the gastrocnemius on vertical jump performance. Journal of Strength Conditioning Research, Connecticut, v.19, n.3, p. 684-688, 2005. Disponível em: <http://journals.Iww.com/nscaiscr/Abstract/2005/08000/Surface Electromyograp hic Assessment of the Effect.33.aspx>. Acesso em: 24 mar. 2010.

WINCHESTER, J.B.; NELSON, A.G.; KOKKONEN, J. A single 30-s stretch is sufficient to inhibit maximal voluntary strength. Research 
Quarterly for Exercise and Sport, Washington, v.80, n.2, p.257-261, 2009. Disponível em: <http://www.ncbi.nlm.nih.gov/pubmed/19650391>. Acesso em: 14 fev. 2011.

WINKE, M.R.; JONES, N.B.; BERGER, C.G.; YATES, J.W. Moderate static stretching and torque production of the knee flexors. Journal of Strength Conditioning Research, Connecticut, v.24, n.3, p.706-710, 2010. Disponível em: $<$ http://journals.lww.com/nscajscr/Fulltext/2010/03000/Moderate Static Stretchi ng and Torque Production.16.aspx>. Acesso em: 27 mar. 2011.

YAMAGUCHI, T.; ISHII, K.; YAMANAKA, M.; YASUDA, K. Acute effect of static stretching on power output during concentric dynamic constant external resistance leg extension. Journal of Strength Conditioning Research, Connecticut, v. 20, n.4, p. 804-810, 2006. Disponível em: $<$ http://journals.Iww.com/nscajscr/Abstract/2006/11000/Acute Effect of Static Stretching on Power Output.13.aspx>. Acesso em: 18 jul. 2011.

YAMAGUCHI, T.; ISHII, K. Effects of static stretching for 30 seconds and dynamic stretching on leg extension power. Journal of Strength

Conditioning Research, Connecticut, v.19, n.3, p.677-683, 2005. Disponível em:

$<$ http://journals.Iww.com/nsca-

jscr/Abstract/2005/08000/Effects of Static Stretc hing for 30 Seconds and.32.aspx>. Acesso em: 18 jul. 2011.

\section{Endereço:}

Anderson Caetano Paulo Universidade Nove de Julho.

Rua Guaranésia, 425 Bairro Vila Maria

São Paulo SP Brasil

02112-000

Telefone/Fax: (11) 2633-9309

e-mail: andersoncaetano@gmail.com

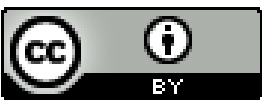

Motriz. Revista de Educação Física. UNESP, Rio Claro, SP, Brasil - elSSN: 1980-6574 - está licenciada sob Creative Commons - Atribuicão 3.0

Recebido em: 27 de maio de 2011. Aceito em: 3 de fevereiro de 2012. 